Pacific

Journal of

Mathematics

SOME CHARACTERIZATIONS OF CAMPANATO SPACES VIA COMMUTATORS ON MORREY SPACES

Shaoguang Shi AND Shanzhen Lu

Volume 264 No. 1

July 2013 


\title{
SOME CHARACTERIZATIONS OF CAMPANATO SPACES VIA COMMUTATORS ON MORREY SPACES
}

\author{
ShaOguang Shi AND Shanzhen LU
}

\begin{abstract}
We give some creative characterizations of Campanato spaces via the boundedness of commutators associated with the Calderón-Zygmund singular integral operator by some new methods instead of the sharp maximal function theorem.
\end{abstract}

\section{Introduction and main results}

Let $-n / p \leq \beta<1$ and $1 \leq p<\infty$. A locally integrable function $f$ is said to belong to the Campanato spaces $C^{p, \beta}\left(\mathbb{R}^{n}\right)$ if

$$
\|f\|_{C^{p, \beta}\left(\mathbb{R}^{n}\right)}=\sup _{Q}\|f\|_{C^{p, \beta}(Q)}:=\sup _{Q} \frac{1}{|Q|^{\beta / n}}\left(\frac{1}{|Q|} \int_{Q}\left|f-f_{Q}\right|^{p} d x\right)^{1 / p}<\infty,
$$

where $f_{Q}=\frac{1}{|Q|} \int_{Q} f(x) d x, Q$ denotes any cube contained in $\mathbb{R}^{n}$ and $|Q|$ is the Lebesgue measure of $Q$.

Campanato spaces are useful tools in the regularity theory of PDEs as a result of their better structures, which allow us to give an integral characterization of the spaces of Hölder continuous functions. This leads to a generalization of the classical Sobolev embedding theorem (see, e.g., [Lemarié-Rieusset 2007; Lu 1995; 1998]). It is also well known that $C^{1,1 / p-1}$ is the dual space of Hardy space $H^{p}\left(\mathbb{R}^{n}\right)$ when $0<p<1$ (see [Triebel 1992]). For a recent account of the theory on $C^{p, \beta}\left(\mathbb{R}^{n}\right)$, we refer the reader to [Duong et al. 2007; Lin et al. 2011; Nakai 2006; Yang et al. 2010].

It's obvious that $\beta=0$ implies $C^{p, 0}\left(\mathbb{R}^{n}\right)=B M O\left(\mathbb{R}^{n}\right)$ with the norm

$$
\|f\|_{B M O\left(\mathbb{R}^{n}\right)}=\sup _{Q} \frac{1}{|Q|} \int_{Q}\left|b-b_{Q}\right| d x .
$$

Corresponding author: Shaoguang Shi. This work was partially supported by NSF of China (Grant Nos. 10931001 and 11271175), NSF of Shandong Province (Grant No. ZR2012AQ026), the Key Laboratory of Mathematics and Complex System (Beijing Normal University), Ministry of Education, China.

MSC2010: primary 42B20; secondary 42B25.

Keywords: Calderón-Zygmund singular integral, commutator, Morrey space, weight. 
When $0<\beta<1$ and $1 \leq p<\infty$, we have $C^{p, \beta}\left(\mathbb{R}^{n}\right)=\operatorname{Lip}_{\beta}\left(\mathbb{R}^{n}\right)$ (see [DeVore and Sharpley 1984; Janson et al. 1983]) with the equivalent norm

$$
\begin{aligned}
\|f\|_{\operatorname{Lip}_{\beta}\left(\mathbb{R}^{n}\right)} & \approx \sup _{Q} \frac{1}{|Q|^{1+\beta / n}} \int_{Q}\left|f-f_{Q}\right| \\
& \approx \sup _{Q}\left(\frac{1}{|Q|^{1+q \beta / n}} \int_{Q}\left|f-f_{Q}\right|^{q}\right)^{1 / q},
\end{aligned}
$$

where $1 \leq q \leq \infty$ and $\operatorname{Lip}_{\beta}\left(\mathbb{R}^{n}\right)$ is the Lipschitz functional space.

When $-n / p \leq \beta<0$, there are several stages in the study of $C^{p, \beta}\left(\mathbb{R}^{n}\right)$. Let $\Omega$ be a connected open set of $\mathbb{R}^{n}$. Denote by $\bar{\Omega}$ the closure of $\Omega$, and by diam $\Omega$ the diameter of $\Omega$. For any $x_{0} \in \mathbb{R}^{n}$ and $l \in(0, \infty)$, set $B\left(x_{0}, l\right)=\left\{x \in \mathbb{R}^{n}:\left|x-x_{0}\right|<l\right\}$ and $\Omega\left(x_{0}, l\right)=B\left(x_{0}, l\right) \cap \Omega$. The following space was first introduced by Morrey [1938] to investigate the local behavior of solutions to the second order elliptic PDE

$$
\|f\|_{M^{p, \beta}(\Omega)}=\sup _{\substack{x_{0} \in \bar{\Omega} \\ l \in(0, \operatorname{diam} \Omega)}} \frac{1}{\left|\Omega\left(x_{0}, l\right)\right|^{\beta / n}}\left(\frac{1}{\left|\Omega\left(x_{0}, l\right)\right|} \int_{\Omega\left(x_{0}, l\right)}|f|^{p}\right)^{1 / p},
$$

where $f \in L_{\text {loc }}^{p}(\Omega), 1 \leq p<\infty$ and $-n / p \leq \beta<0$. When $\Omega=\mathbb{R}^{n}, M^{p, \beta}\left(\mathbb{R}^{n}\right)$ is the classical Morrey space, whose norm is defined by

$$
\|f\|_{M^{p, \beta}\left(\mathbb{R}^{n}\right)}=\sup _{Q}\|f\|_{M^{p, \beta}(Q)}=\sup _{Q} \frac{1}{|Q|^{\beta / n}}\left(\frac{1}{|Q|} \int_{Q}|f|^{p}\right)^{1 / p} .
$$

$M^{p, \beta}\left(\mathbb{R}^{n}\right)$ is an expansion of $L^{p}\left(\mathbb{R}^{n}\right)$ in the sense that $M^{p,-n / p}\left(\mathbb{R}^{n}\right)=L^{p}\left(\mathbb{R}^{n}\right)$. Similarly, for $1 \leq p<\infty,-n / p \leq \beta<0$, a function $f \in L_{\mathrm{loc}}^{p}(\Omega)$ is said to belong to the Campanato space $C^{p, \beta}(\Omega)$ if

$$
\|f\|_{C^{p, \beta}(\Omega)}=\sup _{\substack{x_{0} \in \bar{\Omega} \\ l \in(0, \operatorname{diam} \Omega)}} \frac{1}{\left|\Omega\left(x_{0}, l\right)\right|^{\beta / n}}\left(\frac{1}{\left|\Omega\left(x_{0}, l\right)\right|} \int_{\Omega\left(x_{0}, l\right)}\left|f-f_{\Omega\left(x_{0}, l\right)}\right|^{p}\right)^{1 / p}<\infty .
$$

Campanato [1963] proved that, if $\operatorname{diam} \Omega<\infty$ and there exists a positive constant $C$ such that

$$
\left|\Omega\left(x_{0}, l\right)\right| \geq C l^{n},
$$

for every $x_{0} \in \bar{\Omega}$ and $l \in(0$, diam $\Omega)$, then

$$
M^{p, \beta}(\Omega)=C^{p, \beta}(\Omega) .
$$

(For more accounts about (1-4), see [Rupflin 2008], for example.) Throughout this paper, the letter $C$ stands for a positive constant which may vary from line to line. When diam $\Omega=\infty$ (i.e., $\Omega$ is unbounded, as when $\Omega=\mathbb{R}^{n}$, for example), Sakamoto and Yabuta [1999] pointed out that when $1 \leq p<\infty$ and $\beta \in[-n / p, 0), C^{p, \beta}\left(\mathbb{R}^{n}\right)$ 
is equivalent to $M^{p, \beta}\left(\mathbb{R}^{n}\right)$. But Lin [2009] gave a counterexample to verify that when $1 \leq p<\infty$ and $\beta \in[-n / p, 0)$, we have

$$
M^{p, \beta}\left(\mathbb{R}^{n}\right) \subsetneq C^{p, \beta}\left(\mathbb{R}^{n}\right),
$$

which implies that the statement in [Sakamoto and Yabuta 1999] may be inaccurate. More precisely, on account of the remark above, we have

$$
C^{p, \beta}\left(\mathbb{R}^{n}\right) \begin{cases}=B M O\left(\mathbb{R}^{n}\right) & \text { for } \beta=0, \\ =\operatorname{Lip}_{\beta}\left(\mathbb{R}^{n}\right) & \text { for } 0<\beta<1, \\ \supset M^{p, \beta}\left(\mathbb{R}^{n}\right) & \text { for }-n / p<\beta<0 .\end{cases}
$$

Let $T$ be a linear operator and $b$ a suitable function. For a proper function $f$, the commutator $T_{b}$ is defined by

$$
T_{b}(f):=b T f-T(b f) .
$$

In this paper, we give some characterizations of $C^{p, \beta}\left(\mathbb{R}^{n}\right)$ in terms of the boundedness of $T_{b}$, where $T$ is the Calderón-Zygmund singular integral operator

$$
T f(x)=\text { p.v. } \int_{\mathbb{R}^{n}} K(x-y) f(y) d y ;
$$

here $K \in C^{\infty}\left(S^{n-1}\right)$ is a Calderón-Zygmund kernel that satisfies

$$
K(x)=K(x /|x|) /|x|^{n} \quad \text { for }|x| \neq 0
$$

and

$$
\int_{S^{n-1}} K=0 .
$$

For more on the theory of the Calderón-Zygmund singular integral operator $T$, see [Grafakos 2004; Janson 1978; Lu 2011; Lu et al. 2007; Stein 1970], for example.

There are many classical works about the characterizations of Campanato spaces by the boundedness of $T_{b}$ on Lebesgue spaces. Coifman, Rochberg and Weiss [Coifman et al. 1976] gave a characterization of $B M O\left(\mathbb{R}^{n}\right)$ in terms of the commutator $T_{b}$ :

$$
b \in B M O\left(\mathbb{R}^{n}\right) \Longleftrightarrow T_{b}: L^{p}\left(\mathbb{R}^{n}\right) \rightarrow L^{p}\left(\mathbb{R}^{n}\right) \text { if } 1<p<\infty .
$$

Janson [1978] gave a characterization of $\operatorname{Lip}_{\beta}\left(\mathbb{R}^{n}\right)$ by the $\left(L^{p}, L^{q}\right)$-boundedness of the commutator $T_{b}$ : If $0<\beta<1$, then $b \in \operatorname{Lip}_{\beta}\left(\mathbb{R}^{n}\right) \Longleftrightarrow T_{b}: L^{p}\left(\mathbb{R}^{n}\right) \rightarrow L^{q}\left(\mathbb{R}^{n}\right)$ if $1<p<q<\infty$ and $1 / q=1 / p-\beta / n$.

Paluszyński [1995] gave a new characterization of $\operatorname{Lip}_{\beta}\left(\mathbb{R}^{n}\right)$ by the $\left(L^{p}, \dot{F}_{p, \infty}^{\beta}\right)$ boundedness of the commutator $T_{b}$ : If $0<\beta<1$, then 


$$
b \in \operatorname{Lip}_{\beta}\left(\mathbb{R}^{n}\right) \Longleftrightarrow T_{b}: L^{p}\left(\mathbb{R}^{n}\right) \rightarrow \dot{F}_{p, \infty}^{\beta}\left(\mathbb{R}^{n}\right) \text { if } 1<p<\infty,
$$

where $\dot{F}_{p, \infty}^{\beta}\left(\mathbb{R}^{n}\right)$ is the homogeneous Triebel-Lizorkin space with the equivalent norm

$$
\|f\|_{\dot{F}_{p, \infty}^{\beta}\left(\mathbb{R}^{n}\right)} \approx\left\|\sup _{Q} \frac{1}{|Q|^{1+\beta / n}} \int_{Q}\left|b-b_{Q}\right|\right\|_{L^{p}} .
$$

As a natural extension of Lebesgue space, it is interesting to know whether Campanato spaces can be characterized by the boundedness of $T_{b}$ on Morrey spaces.

Ding [1997] characterized $B M O\left(\mathbb{R}^{n}\right)$ by the $\left(M^{p, \beta}\left(\mathbb{R}^{n}\right), M^{p, \beta}\left(\mathbb{R}^{n}\right)\right)$-boundedness of $T_{b}$ :

$$
b \in B M O\left(\mathbb{R}^{n}\right) \Longleftrightarrow T_{b}: M^{p, \beta}\left(\mathbb{R}^{n}\right) \rightarrow M^{p, \beta}\left(\mathbb{R}^{n}\right) \text { if } 1<p<\infty,-n / p \leq \beta<0 .
$$

In the rest of this paper, we shall establish the characterizations of other cases of Campanato spaces - namely, $\operatorname{Lip}_{\beta}\left(\mathbb{R}^{n}\right)$ for $0<\beta<1$ and $M^{p, \beta}\left(\mathbb{R}^{n}\right)$ for $-n / p \leq$ $\beta<0$ - using certain boundedness properties of $T_{b}$ on Morrey spaces.

Now, we formulate our first result as follows:

Theorem 1.1. Let $1<p<\infty, 0<\alpha<1,-n / p \leq \beta<0,1+p \beta / n<p / q$, $1 / q=1 / p-\alpha / n$ and $\tilde{\beta}=(q-p) / p+q \beta / n$. The following statements are equivalent:

(1) $b \in \operatorname{Lip}_{\alpha}\left(\mathbb{R}^{n}\right)$.

(2) $T_{b}$ is a bounded operator from $M^{p, \beta}\left(\mathbb{R}^{n}\right)$ to $M^{q, \tilde{\beta}}\left(\mathbb{R}^{n}\right)$.

We say that a nonnegative function $f$ belongs to the reverse Hölder class $R H^{r}$ if for any $Q \subset \mathbb{R}^{n}$ and $1<r<\infty$ we have

$$
\left(\frac{1}{|Q|} \int_{Q}|f|^{r} d x\right)^{1 / r} \leq \frac{C}{|Q|} \int_{Q}|f| d x .
$$

When $r=\infty$, we say that $f \in R H^{\infty}$ if $f \in L_{\text {loc }}^{\infty}\left(\mathbb{R}^{n}\right)$ and there exists a constant $C$ such that

$$
\|f\|_{L^{\infty}(Q)}:=\sup _{Q \ni x}|f(x)| \leq \frac{C}{|Q|} \int_{Q}|f| d x .
$$

For $1<r<\infty$, it is easy to see that $R H^{\infty}=\bigcup_{r>1} R H^{r}$. Reverse Hölder classes contain many kinds of functions. For example, if $P(x)$ is a polynomial and $\gamma>0$, then $f(x)=|P(x)|^{\gamma} \in R H^{\infty}$ (see [Fefferman 1983]). (For more theories about $R H^{r}$, see [Cruz-Uribe and Neugebauer 1995; Harboure et al. 1998], for example.)

Theorem 1.2. Assume $\max \{1, n /(1-\beta)\}<p<\infty,-n / p \leq \beta<0,1<p_{i}<\infty$ $(i=1,2), p_{1} \in \mathbb{N}$ even, $-n / p_{i} \leq \beta_{i}<0,1 / p=1 / p_{1}+1 / p_{2}$ and $\beta=\beta_{1}+\beta_{2}$. If $\Omega$ satisfies (1-3) and $\operatorname{diam} \Omega<\infty$, the following statements are equivalent: 
(1) $b \in M^{p_{1}, \beta_{1}}(\Omega)$.

(2) If $b \in R H^{\infty}, T_{b}$ is a bounded operator from $M^{p_{2}, \beta_{2}}(\Omega)$ to $M^{p, \beta}(\Omega)$.

The advantage of using the assumption $\operatorname{diam} \Omega<\infty$ lies in the fact that the equivalent norm of (1-4) is used in the proof of Theorem 1.2. If $\Omega=\mathbb{R}^{n}$, we can obtain the following characterizations of Campanato spaces:

Theorem 1.3. Assume $\max \{1, n /(1-\beta)\}<p<\infty,-n / p \leq \beta<0,1<p_{i}<\infty$ $(i=1,2), p_{1} \in \mathbb{N}$ even, $-n / p_{i} \leq \beta_{i}<0,1 / p=1 / p_{1}+1 / p_{2}$ and $\beta=\beta_{1}+\beta_{2}$. The following statements are equivalent:

(1) $b \in C^{p_{1}, \beta_{1}}\left(\mathbb{R}^{n}\right)$.

(2) $T_{b}$ is a bounded operator from $M^{p_{2}, \beta_{2}}\left(\mathbb{R}^{n}\right)$ to $C^{p, \beta}\left(\mathbb{R}^{n}\right)$ if $b$ further satisfies that there exists a constant $C>0$ such that for any $Q \subset \mathbb{R}^{n}$,

$$
\sup _{Q}\left|b-b_{Q}\right| \leq \frac{C}{|Q|} \int_{Q}\left|b-b_{Q}\right| .
$$

Remark 1. Inequalities (1-8) and (1-9) can be thought of as a form of mean value equality. Besides polynomial functions, mean value equalities also characterize harmonic functions (see [Gilbarg and Trudinger 1983]).

Remark 2. Solutions to a large class of elliptic second order PDEs satisfy the mean value inequality. Therefore, Theorem 1.2 and Theorem 1.3 can give characterizations of the space of solutions to some second order elliptic PDEs. Take Laplace's equation, for example. If $b$ is a solution to the equation

$$
\Delta u=0,
$$

where $\Delta$ is the Laplace operator and $u$ is a function defined on the bounded domain $\Omega \subset \mathbb{R}^{n}$, then $b$ satisfies (1-9); see [Gilbarg and Trudinger 1983, Theorem 2.1]. Therefore, if the commutator $T_{b}$ associated to $b$ is bounded from $M^{p_{2}, \beta_{2}}\left(\mathbb{R}^{n}\right)$ to $C^{p, \beta}\left(\mathbb{R}^{n}\right)$, then the space of solutions to (1-10) is the Campanato space $C^{p_{1}, \beta_{1}}\left(\mathbb{R}^{n}\right)$.

Remark 3. We emphasize that the methods in dealing with $C^{p, \beta}$ when $\beta<0$ are quite different from that of $\beta \geq 0$, and there are essential difficulties in establishing the characterizations of Campanato spaces on Morrey spaces when $\beta<0$. Therefore, we set up Theorem 1.3 under the condition that the symbol of the commutator satisfies the mean value inequality. Condition (1-9) in Theorem 1.3 was intrinsic to the proof of the converse characterizations of $C^{p_{1}, \beta_{1}}\left(\mathbb{R}^{n}\right)$. Of course, there are essential differences between the ideas in the proof of Theorem 1.2 and Theorem 1.3 and that of [Janson 1978] and [Paluszyński 1995], where the sharp maximal function theorem were used.

Our theorems provide natural and intrinsic characterizations of Campanato spaces on Morrey spaces. It is also worth pointing out that our paper is the first work 
on the problem of commutators whose symbol belongs to Morrey spaces. Our viewpoints will shed some new lights on characterizations of Campanato spaces via commutators formed by other operators on Morrey spaces, such as fractional integrals, oscillatory integral operators and Hardy-Littlewood-Paley operators. Besides Euclidean space, characterizations of Campanato spaces on other spaces can similarly be considered, such as on homogeneous groups. Partly inspired by [Janson 1978] and [Paluszyński 1995], we prove Theorems 1.1-1.3 in Section 2.

\section{Proof of the main results}

For the proofs we need some lemmas about the estimates of operators on Morrey spaces.

Lemma 2.1 [Chiarenza and Frasca 1987]. Let $1<p<n / \alpha, 0<\alpha<n, 1 / q=$ $1 / p-\alpha / n, 0<1+p \beta / n<p / q,-n / p \leq \beta<0$ and $\tilde{\beta}=(q-p) / p+q \beta / n$. Then the fractional integral operator

$$
I_{\alpha} f(x)=\int_{\mathbb{R}^{n}} \frac{f(y)}{|x-y|^{n-\alpha}} d y
$$

is bounded from $M^{p, \beta}\left(\mathbb{R}^{n}\right)$ to $M^{q, \tilde{\beta}}\left(\mathbb{R}^{n}\right)$.

Lemma 2.2 [Komori and Shirai 2009]. Let $1<p<\infty$ and $-n / p \leq \beta<0$. Then $T$ is bounded from $M^{p, \beta}\left(\mathbb{R}^{n}\right)$ to $M^{p, \beta}\left(\mathbb{R}^{n}\right)$.

Proof of Theorem 1.1. (1) $\Rightarrow$ (2). Together, (1-2) and (1-6) imply

$$
\begin{aligned}
\left|T_{b} f(x)\right| & \leq \int_{\mathbb{R}^{n}}|b(x)-b(y)||K(x-y)||f(y)| d y \\
& \leq \int_{\mathbb{R}^{n}} \frac{|f(y)|}{|x-y|^{n-\alpha}} d y \leq I_{\alpha}(|f(x)|) .
\end{aligned}
$$

Therefore, $T_{b}$ is bounded from $M^{p, \beta}\left(\mathbb{R}^{n}\right)$ to $M^{q, \tilde{\beta}}\left(\mathbb{R}^{n}\right)$ by Lemma 2.1 .

$(2) \Rightarrow(1)$. The proof consists of the construction of a proper commutator. We follow [Janson 1978] in choosing $z_{0} \neq 0$ and $\delta>0$ such that $1 / K(z)$ can be expressed in the neighborhood $\left|z-z_{0}\right|<\sqrt{n} \delta$ as the absolute convergent Fourier series

$$
\frac{1}{K(z)}=\sum a_{n} e^{i v_{n} \cdot z}
$$

where the exact form of the vectors $v_{n}$ is irrelevant. Set $z_{1}=\delta^{-1} z_{0}$. If $\left|z-z_{1}\right|<\sqrt{n}$, it follows from (1-6) that

$$
\frac{1}{K(z)}=\frac{\delta^{-n}}{K(\delta z)}=\delta^{-n} \sum a_{n} e^{i v_{n} \cdot \delta z} .
$$

Choose now any cube $Q=Q\left(x_{0}, r\right)$. Set $y_{0}=x_{0}-r z_{1}$ and $Q^{\prime}=Q\left(y_{0}, r\right)$. Thus, 
if $x \in Q$ and $y \in Q^{\prime}$,

$$
\left|\frac{x-y}{r}-z_{1}\right| \leq\left|\frac{x-x_{0}}{r}-\frac{y-y_{0}}{r}\right| \leq \sqrt{n} .
$$

Denoting $s(x)=\operatorname{sgn}\left(b(x)-b_{Q^{\prime}}\right)$, by (2-1) we have

$$
\begin{aligned}
\int_{Q}\left|b(x)-b_{Q^{\prime}}\right| d x & =\int_{Q}\left(b(x)-b_{Q^{\prime}}\right) s(x) d x \\
& =\frac{1}{\left|Q^{\prime}\right|} \int_{Q} \int_{Q^{\prime}}(b(x)-b(y)) s(x) d y d x \\
& =\frac{1}{r^{n}} \iint_{\mathbb{R}^{n}}(b(x)-b(y)) s(x) \frac{r^{n} K(x-y)}{K((x-y) / r)} \chi_{Q}(x) \chi_{Q^{\prime}}(y) d y d x \\
& =C \sum a_{n} \iint_{\mathbb{R}^{n}}(b(x)-b(y)) K(x-y) e^{i(\delta / r) v_{n} \cdot x} \\
& \times s(x) \chi_{Q}(x) e^{-i(\delta / r) v_{n} \cdot y} \chi_{Q^{\prime}}(y) d y d x .
\end{aligned}
$$

Taking

$$
g_{n}(y)=e^{-i(\delta / r) v_{n} \cdot y} \chi_{Q^{\prime}}(y) \quad \text { and } \quad h_{n}(x)=e^{i(\delta / r) v_{n} \cdot x} s(x) \chi_{Q}(x),
$$

we obtain

$$
\begin{aligned}
\int_{Q}\left|b(x)-b_{Q^{\prime}}\right| d x & =C \sum a_{n} \iint_{\mathbb{R}^{n}}(b(x)-b(y)) K(x-y) g_{n}(y) h_{n}(x) d y d x \\
& =C \sum a_{n} \int_{\mathbb{R}^{n}} T_{b} g_{n}(x) h_{n}(x) d x \\
& \leq C \sum\left|a_{n}\right| \int_{\mathbb{R}^{n}}\left|T_{b} g_{n}(x)\right|\left|h_{n}(x)\right| d x \\
& \leq C \sum\left|a_{n}\right| \int_{\mathbb{R}^{n}}\left|T_{b} g_{n}(x)\right| d x .
\end{aligned}
$$

Applying the Hölder inequality to $\int_{\mathbb{R}^{n}}\left|T_{b} g_{n}(x)\right| d x$, we have

$$
\begin{aligned}
\int_{\mathbb{R}^{n}}\left|T_{b} g_{n}(x)\right| d x & \leq|Q|^{1+(\alpha+\beta) / n}\left\|T_{b} g_{n}\right\|_{M^{q, \tilde{\beta}}\left(\mathbb{R}^{n}\right)} \\
& \leq C|Q|^{1+(\alpha+\beta) / n}\left\|g_{n}\right\|_{M^{p, \beta}\left(\mathbb{R}^{n}\right)} \\
& \leq C|Q|^{1+\alpha / n},
\end{aligned}
$$

since $\left\|g_{n}\right\|_{M^{p, \beta}\left(\mathbb{R}^{n}\right)}=|Q|^{-\beta / n}$. Thus we have obtained

$$
\frac{1}{|Q|^{1+\alpha / n}} \int_{Q}\left|b(x)-b_{Q^{\prime}}\right| d x \leq C,
$$

which completes the proof of Theorem 1.1 by (1-2). 
Theorem 1.2 is a restatement of Theorem 1.3 when $C^{p, \beta}$ spaces over domains with finite volume, so we give the proof of Theorem 1.3 first. Again, we begin with some lemmas that are essential to our analysis.

Lemma 2.3. Let $p, p_{1}, p_{2}, \beta, \beta_{1}, \beta_{2}$ and $b$ be the same as in Theorem 1.3. Then

$$
\left\|\left(b-b_{Q}\right) f \chi_{Q}\right\|_{L^{p}\left(\mathbb{R}^{n}\right)} \leq|Q|^{1 / p+\beta / n}\|b\|_{C^{p_{1}, \beta_{1}\left(\mathbb{R}^{n}\right)}}\|f\|_{M^{p_{2}, \beta_{2}\left(\mathbb{R}^{n}\right)}} .
$$

This follows from Hölder's inequality:

$$
\begin{aligned}
\left\|\left(b-b_{Q}\right) f \chi_{Q}\right\|_{L^{p}\left(\mathbb{R}^{n}\right)} & \leq\left(\int_{Q}\left|b-b_{Q}\right|^{p_{1}}\right)^{1 / p_{1}}\left(\int_{Q}|f|^{p_{2}}\right)^{1 / p_{2}} \\
& \leq|Q|^{1 / p(1+p \beta / n)}\|b\|_{C^{p_{1}, \beta_{1}}\left(\mathbb{R}^{n}\right)}\|f\|_{M^{p_{2}, \beta_{2}}\left(\mathbb{R}^{n}\right)} .
\end{aligned}
$$

Lemma 2.4. Suppose that $Q_{*} \subset Q$ and $b \in C^{p_{1}, \beta_{1}}\left(\mathbb{R}^{n}\right)$ with $1<p_{1}<\infty$ and $-n / p_{1} \leq \beta_{1}<0$. Then the following estimate holds:

$$
\left|b_{Q_{*}}-b_{Q}\right| \leq C\|b\|_{C^{p_{1}, \beta_{1}\left(\mathbb{R}^{n}\right)}}\left|Q_{*}\right|^{\beta_{1} / n} .
$$

We divide the proof into two cases.

Case 1: Suppose $Q_{*} \subset Q \subseteq 2 Q_{*}$. Hölder's inequality yields

$$
\begin{aligned}
\left|b_{Q_{*}}-b_{Q}\right| & \leq \frac{1}{\left|Q_{*}\right|} \int_{Q_{*}}\left|b-b_{Q}\right|+\frac{1}{|Q|} \int_{Q}\left|b-b_{Q}\right| \\
& \leq C \frac{1}{|Q|} \int_{Q}\left|b-b_{Q}\right| \leq C\left(\int_{Q}\left|b-b_{Q}\right|^{p_{1}}\right)^{1 / p_{1}}|Q|^{-1 / p_{1}} \\
& \leq C\|b\|_{C^{p_{1}, \beta_{1}\left(\mathbb{R}^{n}\right)}\left|Q_{*}\right|^{\beta_{1} / n}}
\end{aligned}
$$

Case 2: Suppose $2 Q_{*} \subset Q$. Choose a sequence of nested cubes

$$
Q_{*}=: Q_{1} \subset Q_{2} \subset \cdots Q=: Q_{m+1},
$$

with $\left|Q_{i+1}\right|=2^{n}\left|Q_{i}\right|$ for $1 \leq i \leq m$. By the results of Case 1 , we have

$$
\begin{aligned}
\left|b_{Q_{*}}-b_{Q}\right| & =\left|b_{Q_{1}}-b_{Q_{2}}+b_{Q_{2}}-\cdots+b_{Q_{m}}-b_{Q_{m+1}}\right| \\
& \leq \sum_{i=1}^{m}\left|b_{Q_{i}}-b_{Q_{i+1}}\right| \leq\|b\|_{C^{p_{1}, \beta_{1}}\left(\mathbb{R}^{n}\right)} \sum_{i=1}^{m} 2^{(i+1) \beta_{1}}\left|Q_{*}\right|^{\beta_{1} / n} \\
& \leq C\|b\|_{C^{p_{1}, \beta_{1}\left(\mathbb{R}^{n}\right)}\left|Q_{*}\right|^{\beta_{1} / n}}
\end{aligned}
$$

which is (2-2).

The following imbedding theorem for $L^{p}$ spaces over domains with finite volume is very useful in the analysis of inequality, which you can find in any book about Sobolev spaces (see [Adams and Fournier 2003], for example). 
Lemma 2.5. Suppose that $|\Omega|=\int_{\Omega} 1 d x<\infty$ and $1 \leq p \leq q \leq \infty$. If $f \in L^{q}(\Omega)$, then $f \in L^{p}(\Omega)$ and

$$
\|f\|_{L^{p}(\Omega)} \leq C|\Omega|^{1 / p-1 / q}\|f\|_{L^{q}(\Omega)} .
$$

Proof of Theorem 1.3. (1) $\Rightarrow$ (2). For a cube $Q=Q\left(x_{Q}, r\right) \subset \mathbb{R}^{n}$ and $y \in Q$, take $f \in M^{p_{2}, \beta_{2}}\left(\mathbb{R}^{n}\right)$ and set $f_{1}=f \chi_{2 Q}$ and $f_{2}=f-f_{1}$. After noticing that

$$
T_{b} f=T_{\left(b-b_{Q}\right)} f,
$$

we have

$$
\begin{aligned}
\left(\frac{1}{|Q|^{1+p \beta / n}} \int_{Q}\left|T_{b} f-\left(T_{b} f\right)_{Q}\right|^{p}\right)^{1 / p} & \\
& =\left(\frac{1}{|Q|^{1+p \beta / n}} \int_{Q}\left|T_{\left(b-b_{Q}\right)} f-\left(T_{\left(b-b_{Q}\right)} f\right)_{Q}\right|^{p}\right)^{1 / p} \\
& \leq\left(\frac{1}{|Q|^{1+p \beta / n}} \int_{Q}\left|T_{\left(b-b_{Q}\right)} f-\left(b-b_{Q}\right) f_{2}\left(x_{Q}\right)\right|^{p}\right)^{1 / p} \\
& \leq I+I I+I I I,
\end{aligned}
$$

where

$$
\begin{aligned}
I & :=\left(\frac{1}{|Q|^{1+p \beta / n}} \int_{Q}\left|\left(b-b_{Q}\right) T f\right|^{p}\right)^{1 / p}, \\
I I & :=\left(\frac{1}{|Q|^{1+p \beta / n}} \int_{Q}\left|T\left(b-b_{Q}\right) f_{1}\right|^{p}\right)^{1 / p}, \\
I I I & :=\left(\frac{1}{|Q|^{1+p \beta / n}} \int_{Q}\left|\left(T\left(b-b_{Q}\right) f_{2}\right)(y)-\left(T\left(b-b_{Q}\right) f_{2}\right)\left(x_{Q}\right)\right|^{p}\right)^{1 / p} .
\end{aligned}
$$

Hölder's inequality and Lemma 2.2 imply

$$
\begin{aligned}
I & =\frac{1}{|Q|^{1 / p+\beta / n}}\left(\int_{Q}\left|\left(b-b_{Q}\right) T f\right|^{p}\right)^{1 / p} \\
& \leq \frac{1}{|Q|^{1 / p+\beta / n}}\left(\int_{Q}\left|b-b_{Q}\right|^{p_{1}}\right)^{1 / p_{1}}\left(\int_{Q}|T f|^{p_{2}}\right)^{1 / p_{2}} \\
& \leq C\|b\|_{C^{p_{1}, \beta_{1}\left(\mathbb{R}^{n}\right)}}\|T f\|_{M^{p_{2}, \beta_{2}\left(\mathbb{R}^{n}\right)}} \\
& \leq C\|b\|_{C^{p_{1}, \beta_{1}\left(\mathbb{R}^{n}\right)}}\|f\|_{M^{p_{2}, \beta_{2}}\left(\mathbb{R}^{n}\right)} .
\end{aligned}
$$

From Lemma 2.3, it follows that

$$
I I \leq \frac{1}{|Q|^{1 / p+\beta / n}}\left\|\left(b-b_{Q}\right) f_{1}\right\|_{L^{p}} \leq C\|b\|_{C^{p_{1}, \beta_{1}}\left(\mathbb{R}^{n}\right)}\|f\|_{M^{p_{2}, \beta_{2}\left(\mathbb{R}^{n}\right)}} .
$$


We now turn to the estimate for the term III. From (1-6), it may be concluded that

$$
\begin{aligned}
\mid\left(T\left(b-b_{Q}\right) f_{2}\right) & (y)-T\left(\left(b-b_{Q}\right) f_{2}\right)\left(x_{Q}\right) \mid \\
& =\left|\int_{R^{n}}\left(K(y-z)-K\left(x_{Q}-z\right)\right)\left(b(z)-b_{Q}\right) f_{2}(z) d z\right| \\
& \leq C \int_{(2 Q)^{c}} \frac{\left|y-x_{Q}\right|}{\left|z-x_{Q}\right|^{n+1}}\left|b(z)-b_{Q}\right||f(z)| d z \\
& \leq C \sum_{k=2}^{\infty} \int_{2^{k} Q \backslash 2^{k-1} Q} \frac{1}{2^{k}\left|2^{k} Q\right|}\left(\left|b(z)-b_{2^{k} Q}\right|+\left|b_{Q}-b_{2^{k} Q}\right|\right)|f(z)| d z \\
& \leq C \sum_{k=2}^{\infty} \frac{1}{2^{k}\left|2^{k} Q\right|} \int_{2^{k} Q}\left(\left|b(z)-b_{2^{k} Q}\right|+\left|b_{Q}-b_{2^{k} Q}\right|\right)|f(z)| d z,
\end{aligned}
$$

which yields

$$
\begin{aligned}
I I I \leq & \left(\frac{1}{|Q|^{1+p \beta / n}} \int_{Q}\left|\sum_{k=2}^{\infty} \frac{1}{2^{k}\left|2^{k} Q\right|} \int_{2^{k} Q}\right| b(z)-b_{2^{k}}|| f(z)|d z|^{p}\right)^{1 / p} \\
& +\left(\frac{1}{|Q|^{1+p \beta / n}} \int_{Q}\left|\sum_{k=2}^{\infty} \frac{1}{2^{k}\left|2^{k} Q\right|} \int_{2^{k} Q}\right| b_{Q}-b_{2^{k} Q}|| f(z)|d z|^{p}\right)^{1 / p} \\
= & : I I I_{1}+I I I_{2} .
\end{aligned}
$$

With repeated application of Lemma 2.3 and the $L^{p}$-boundedness of the HardyLittlewood maximal operator $M$, we can obtain

$$
\begin{aligned}
& I I I_{1} \leq \frac{1}{|Q|^{1 / p+\beta / n}}\left\|\sum_{k=2}^{\infty} \frac{1}{2^{k}\left|2^{k} Q\right|} \int_{2^{k} Q}\left|b(z)-b_{2^{k}} Q\right||f(z)| d z\right\|_{L^{p}} \\
& \leq \frac{1}{|Q|^{1 / p+\beta / n}} \sum_{k=2}^{\infty} \frac{1}{2^{k}}\left\|\frac{1}{\left|2^{k} Q\right|} \int_{2^{k} Q}\left|b(z)-b_{2^{k} Q}\right||f(z)| d z\right\|_{L^{p}} \\
& \leq \sum_{k=2}^{\infty} \frac{1}{2^{k}} \frac{1}{|Q|^{1 / p+\beta / n}}\left\|M\left(\left|b-b_{2^{k} Q}\right||f|\right)\right\|_{L^{p}} \\
& \leq \sum_{k=2}^{\infty} \frac{1}{2^{k}} \frac{1}{|Q|^{1 / p+\beta / n}}\left\|\left|b-b_{2^{k} Q}\right||f|\right\|_{L^{p}} \\
& \leq \sum_{k=2}^{\infty} \frac{1}{2^{k(1-n / p-\beta)}}\|b\|_{C^{p_{1}, \beta_{1}\left(\mathbb{R}^{n}\right)}}\|f\|_{M^{p_{2}, \beta_{2}\left(\mathbb{R}^{n}\right)}} \\
& \leq C\|b\|_{C^{p_{1}, \beta_{1}\left(\mathbb{R}^{n}\right)}}\|f\|_{M^{p_{2}, \beta_{2}\left(\mathbb{R}^{n}\right)}} .
\end{aligned}
$$


Applying Lemma 2.4 and Lemma 2.5 to $\mathrm{III}_{2}$, we have

$$
\begin{aligned}
& I I I_{2} \leq \sum_{k=2}^{\infty} \frac{1}{2^{k}}\left(\frac{1}{|Q|^{1+p \beta / n}} \int_{Q}\left|\frac{1}{\left|2^{k} Q\right|} \int_{2^{k} Q}\right| b_{Q}-b_{2^{k}}|| f(z)|d z|^{p} d x\right)^{1 / p} \\
& \leq \sum_{k=2}^{\infty} \frac{1}{2^{k}}\|b\|_{C^{p_{1}, \beta_{1}\left(\mathbb{R}^{n}\right)}}\left(\frac{|Q|^{\beta_{1} / n}}{|Q|^{1+p \beta / n}} \int_{Q}\left|\frac{1}{\left|2^{k} Q\right|} \int_{2^{k} Q}\right| f(z)|d z|^{p} d x\right)^{1 / p} \\
& \leq \sum_{k=2}^{\infty} \frac{1}{2^{k}}\|b\|_{C^{p_{1}, \beta_{1}\left(\mathbb{R}^{n}\right)}} \frac{1}{|Q|^{1 / p+\beta_{2} / n}}\|M(|f|)\|_{L^{p}} \\
& \leq \sum_{k=2}^{\infty} \frac{1}{2^{k}}\|b\|_{C^{p_{1}, \beta_{1}\left(\mathbb{R}^{n}\right)}} \frac{1}{|Q|^{1 / p+\beta_{2} / n}}\|f\|_{L^{p}} \\
& \leq \sum_{k=2}^{\infty} \frac{1}{2^{k\left(1-n / p-\beta_{2}\right)}}\|b\|_{C^{p_{1}, \beta_{1}}}\|f\|_{M^{p_{2}, \beta_{2}}} \\
& \leq C\|b\|_{C^{p_{1}, \beta_{1}\left(\mathbb{R}^{n}\right)}}\|f\|_{M^{p_{2}, \beta_{2}\left(\mathbb{R}^{n}\right)}} .
\end{aligned}
$$

Thus, we have obtained $I I I \leq C\|b\|_{C^{p_{1}, \beta_{1}\left(\mathbb{R}^{n}\right)}}\|f\|_{M^{p_{2}, \beta_{2}\left(\mathbb{R}^{n}\right)}}$. Our proof ends with the definition of $\|\cdot\|_{C^{p, \beta}\left(\mathbb{R}^{n}\right)}$.

(2) $\Rightarrow(1)$. We first claim that for fixed $Q \subset \mathbb{R}^{n}, b \in C^{p_{1}, \beta_{1}}(Q)$ and $f \in M^{p_{2}, \beta_{2}}(Q)$ with $\|f\|_{M^{p_{2}, \beta_{2}(Q)}}=|Q|^{-\beta_{2} / n}$, we have

$$
\left\|T_{b}^{m} f\right\|_{C^{p, \beta}(Q)} \leq C|Q|^{\beta_{1}(m-1) / n}\|f\|_{M^{p_{2}, \beta_{2}}(Q)} \leq C|Q|^{\left(\beta_{1} m-\beta\right) / n},
$$

where $T_{b}^{m}$ is the $m$-th $\left(m \in Z^{+}\right)$commutator defined by

$$
T_{b}^{m} f(x)=\text { p.v. } \int(b(x)-b(y))^{m} K(x-y) f(y) d y .
$$

We shall prove (2-3) by induction. The case $m=1$ is trivial. We now assume that for any $b \in C^{p_{1}, \beta_{1}}(Q)$, we have

$$
\left\|T_{b}^{m-1} f\right\|_{C^{p, \beta}(Q)} \leq C|Q|^{\beta_{1}(m-2) / n}\|f\|_{M^{p_{2}, \beta_{2}}(Q)} \leq C|Q|^{\left(\beta_{1}(m-1)-\beta\right) / n} .
$$

Next, we show the case $m$. We now observe that

$$
\begin{aligned}
\left|T_{b}^{m} f(x)\right|= & \left|\int(b(x)-b(y))^{m-1} K(x-y) f(y)(b(x)-b(y)) d y\right| \\
\leq & \left|\int(b(x)-b(y))^{m-1} K(x-y) f(y)\left(b(x)-b_{Q}\right) d y\right| \\
& +\left|\int(b(x)-b(y))^{m-1} K(x-y) f(y)\left(b(y)-b_{Q}\right) d y\right| \\
\leq & \left(\left|b-b_{Q}\right|\left|T_{b}^{m-1} f\right|\right)(x)+\left|T_{b}^{m-1}\left(\left(b-b_{Q}\right) f\right)(x)\right|=: J_{1}+J_{2} .
\end{aligned}
$$


Equation (2-4) enables us to estimate $J_{1}$ as

$$
\begin{aligned}
\left\|J_{1}\right\|_{C^{p, \beta}(Q)} & \leq\left\|\left|b-b_{Q}\left\|T_{b}^{m-1} f \mid\right\|_{C^{p, \beta}(Q)}\right.\right. \\
& \leq\left\|b-b_{Q}\right\|_{L^{\infty}}\left\|T_{b}^{m-1} f\right\|_{C^{p, \beta}(Q)} \\
& \leq \frac{1}{|Q|} \int_{Q}\left|b-b_{Q}\right| d x|Q|^{\beta_{1}(m-2) / n}\|f\|_{M^{p_{2}, \beta_{2}(Q)}} \\
& \leq C|Q|^{\beta_{1}(m-1) / n}\|f\|_{M^{p_{2}, \beta_{2}}(Q)} \leq C|Q|^{\left(\beta_{1} m-\beta\right) / n} .
\end{aligned}
$$

With repeated application of (2-4), we have

$$
\begin{aligned}
\left\|J_{2}\right\|_{C^{p, \beta}(Q)} & =\left\|T_{b}^{m-1}\left(\left(b-b_{Q}\right) f\right)\right\|_{C^{p, \beta}(Q)} \\
& \leq C|Q|^{\beta_{1}(m-2) / n}\left\|\left(b-b_{Q}\right) f\right\|_{M^{p_{2}, \beta_{2}}(Q)} \\
& \leq C|Q|^{\beta_{1}(m-2) / n}\left\|b-b_{Q}\right\|_{L^{\infty}}\|f\|_{M^{p_{2}, \beta_{2}}(Q)} \\
& \leq C|Q|^{\beta_{1}(m-1) / n}\|b\|_{C^{p_{1}, \beta_{1}\left(\mathbb{R}^{n}\right)}\|f\|_{M^{p_{2}, \beta_{2}}(Q)} .} \\
& \leq C|Q|^{\left(\beta_{1} m-\beta\right) / n} .
\end{aligned}
$$

We come back to the proof of $(2) \Rightarrow(1)$. The rest of the proof proceeds similarly to that of Theorem 1.1. We apply the same argument again with $s(x)$ replaced by $\operatorname{sgn}\left(b(x)-b_{Q}\right)^{p_{1}}$ to obtain

$$
\int_{Q}\left|b-b_{Q}\right|^{p_{1}} d x \leq C \sum a_{n} \int_{Q}\left|T_{b}^{p_{1}}\left(g_{n}\right)\right| d x .
$$

Combining (2-3) and observing $g_{n} \in M^{p_{2}, \beta_{2}}(Q)$ with the norm $\left\|g_{n}\right\|_{M^{p_{2}, \beta_{2}}(Q)}=$ $|Q|^{-\beta_{2} / n}$, we estimate (2-5) as

$$
\begin{aligned}
\sum a_{n} \int_{Q}\left|T_{b}^{p_{1}}\left(g_{n}\right)\right| d x & \leq C|Q|^{1+\beta / n}\left\|T_{b}^{p_{1}}\left(g_{n}\right)\right\|_{C^{p, \beta}(Q)} \\
& \leq C|Q|^{1+p_{1} \beta_{1} / n+\beta_{2} / n}\left\|g_{n}\right\|_{M^{p_{2}, \beta_{2}}(Q)} \\
& \leq C|Q|^{1+p_{1} \beta_{1} / n} .
\end{aligned}
$$

and then take the supremum of $Q$, completing the proof of Theorem 1.3.

Proof of Theorem 1.2. This proof can be handled in much the same way as that of Theorem 1.3, using (1-5) and replacing $b-b_{Q}$ by $b$ in the proof of (2-3).

\section{Acknowledgements}

The authors thank the anonymous referees cordially for their valuable suggestions on this paper. 


\section{References}

[Adams and Fournier 2003] R. A. Adams and J. J. F. Fournier, Sobolev spaces, 2nd ed., Pure and Applied Mathematics 140, Elsevier/Academic Press, Amsterdam, 2003. MR 2009e:46025 Zbl 1098.46001

[Campanato 1963] S. Campanato, "Proprietà di hölderianità di alcune classi di funzioni", Ann. Scuola Norm. Sup. Pisa (3) 17:1-2 (1963), 175-188. MR 27 \#6119 Zbl 0121.29201

[Chiarenza and Frasca 1987] F. Chiarenza and M. Frasca, "Morrey spaces and Hardy-Littlewood maximal function”, Rend. Mat. Appl. (7) 7:3-4 (1987), 273-279. MR 90f:42017 Zbl 0717.42023

[Coifman et al. 1976] R. R. Coifman, R. Rochberg, and G. Weiss, "Factorization theorems for Hardy spaces in several variables", Ann. of Math. (2) 103:3 (1976), 611-635. MR 54 \#843 Zbl 0326.32011

[Cruz-Uribe and Neugebauer 1995] D. Cruz-Uribe and C. J. Neugebauer, "The structure of the reverse Hölder classes”, Trans. Amer. Math. Soc. 347:8 (1995), 2941-2960. MR 95m:42026 Zbl 0851.42016

[DeVore and Sharpley 1984] R. A. DeVore and R. C. Sharpley, "Maximal functions measuring smoothness”, Mem. Amer. Math. Soc. 47:293 (1984), viii+115. MR 85g:46039 Zbl 0529.42005

[Ding 1997] Y. Ding, "A characterization of BMO via commutators for some operators", Northeast. Math. J. 13:4 (1997), 422-432. MR 2000d:42006 Zbl 0903.42005

[Duong et al. 2007] X. T. Duong, J. Xiao, and L. Yan, "Old and new Morrey spaces with heat kernel bounds", J. Fourier Anal. Appl. 13:1 (2007), 87-111. MR 2007k:42027 Zbl 1133.42017

[Fefferman 1983] C. L. Fefferman, "The uncertainty principle”, Bull. Amer. Math. Soc. (N.S.) 9:2 (1983), 129-206. MR 85f:35001 Zbl 0526.35080

[Gilbarg and Trudinger 1983] D. Gilbarg and N. S. Trudinger, Elliptic partial differential equations of second order, 2nd ed., Grundlehren der Mathematischen Wissenschaften 224, Springer, Berlin, 1983. MR 86c:35035 Zbl 0562.35001

[Grafakos 2004] L. Grafakos, Classical and modern Fourier analysis, Pearson Education, Upper Saddle River, NJ, 2004. MR 2449250 Zbl 1148.42001

[Harboure et al. 1998] E. Harboure, O. Salinas, and B. Viviani, "Reverse-Hölder classes in the Orlicz spaces setting”, Studia Math. 130:3 (1998), 245-261. MR 2000c:42023 Zbl 0905.42012

[Janson 1978] S. Janson, "Mean oscillation and commutators of singular integral operators", Ark. Mat. 16:2 (1978), 263-270. MR 80j:42034 Zbl 0404.42013

[Janson et al. 1983] S. Janson, M. Taibleson, and G. Weiss, "Elementary characterizations of the Morrey-Campanato spaces", pp. 101-114 in Harmonic analysis (Cortona, 1982), edited by G. Mauceri et al., Lecture Notes in Math. 992, Springer, Berlin, 1983. MR 85k:46033 Zbl 0521.46022

[Komori and Shirai 2009] Y. Komori and S. Shirai, "Weighted Morrey spaces and a singular integral operator", Math. Nachr. 282:2 (2009), 219-231. MR 2010h:42019 Zbl 1160.42008

[Lemarié-Rieusset 2007] P. G. Lemarié-Rieusset, "The Navier-Stokes equations in the critical MorreyCampanato space”, Rev. Mat. Iberoam. 23:3 (2007), 897-930. MR 2009g:35221 Zbl 1227.35230

[Lin 2009] H. B. Lin, “Some remarks on Morrey spaces”, Beijing Shifan Daxue Xuebao 45:2 (2009), 111-116. MR 2010f:42042 Zbl 1212.42036

[Lin et al. 2011] H. B. Lin, E. Nakai, and D. Yang, "Boundedness of Lusin-area and $g_{\lambda}^{*}$ functions on localized Morrey-Campanato spaces over doubling metric measure spaces", J. Funct. Spaces Appl. 9:3 (2011), 245-282. MR 2012k:42047 Zbl 1231.42017

[Lu 1995] G. Lu, "Embedding theorems on Campanato-Morrey spaces for vector fields and applications”, C. R. Acad. Sci. Paris Sér. I Math. 320:4 (1995), 429-434. MR 95k:46051 Zbl 0842.46019 
[Lu 1998] G. Lu, "Embedding theorems on Campanato-Morrey spaces for vector fields on Hörmander type", Approx. Theory Appl. (N.S.) 14:1 (1998), 69-80. MR 99h:46053 Zbl 0916.46026

[Lu 2011] S. Lu, "Some results and problems on commutators", Front. Math. China 6:5 (2011), 821-833. MR 2012h:42024 Zbl 1227.42016

[Lu et al. 2007] S. Lu, Y. Ding, and D. Yan, Singular integrals and related topics, World Scientific, Hackensack, NJ, 2007. MR 2009g:42023 Zbl 1124.42011

[Morrey 1938] C. B. Morrey, Jr., "On the solutions of quasi-linear elliptic partial differential equations", Trans. Amer. Math. Soc. 43:1 (1938), 126-166. MR 1501936 Zbl 0018.40501

[Nakai 2006] E. Nakai, "The Campanato, Morrey and Hölder spaces on spaces of homogeneous type”, Studia Math. 176:1 (2006), 1-19. MR 2007j:46054 Zbl 1121.46031

[Paluszyński 1995] M. Paluszyński, "Characterization of the Besov spaces via the commutator operator of Coifman, Rochberg and Weiss", Indiana Univ. Math. J. 44:1 (1995), 1-17. MR 96j:42006 Zbl 0838.42006

[Rupflin 2008] M. Rupflin, "What is a... Morrey Space? What is a... Campanato Space?", preprint, Zürich Graduate School in Mathematics, 2008, http://www.zgsm.ch/fileadmin/math/store/fs08/2902/ Morrey2.pdf.

[Sakamoto and Yabuta 1999] M. Sakamoto and K. Yabuta, "Boundedness of Marcinkiewicz functions”, Studia Math. 135:2 (1999), 103-142. MR 2000g:42027 Zbl 0930.42009

[Stein 1970] E. M. Stein, Singular integrals and differentiability properties of functions, Princeton Mathematical Series 30, Princeton University Press, Princeton, NJ, 1970. MR 44 \#7280 Zbl 0207.13501

[Triebel 1992] H. Triebel, Theory of function spaces, II, Monographs in Mathematics 84, Birkhäuser, Basel, 1992. MR 93f:46029 Zbl 0763.46025

[Yang et al. 2010] D. Yang, D. Yang, and Y. Zhou, "Localized Morrey-Campanato spaces on metric measure spaces and applications to Schrödinger operators", Nagoya Math. J. 198 (2010), 77-119. MR 2012a:46060 Zbl 1214.46019

Received May 5, 2012. Revised December 19, 2012.

\section{ShaOguANG SHI}

DEPARTMENT OF MATHEMATICS

LINYI UNIVERSITY

LINYI 276005

CHINA

AND

SCHOol of Mathematical ScIENCES

BEIJING NORMAL UNIVERSITY

BEIJING, 100875

shishaoguang@mail.bnu.edu.cn

SHANZHEN LU

SCHOOL of Mathematical SCIENCES

BEIJING NORMAL UNIVERSITY

BEIJING, 100875

CHINA

lusz@bnu.edu.cn 


\title{
PACIFIC JOURNAL OF MATHEMATICS
}

\author{
msp.org/pjm
}

Founded in 1951 by E. F. Beckenbach (1906-1982) and F. Wolf (1904-1989)

\section{EDITORS}

V. S. Varadarajan (Managing Editor)

Department of Mathematics

University of California

Los Angeles, CA 90095-1555

pacific@math.ucla.edu

Paul Balmer

Department of Mathematics

University of California

Los Angeles, CA 90095-1555

balmer@math.ucla.edu

Daryl Cooper

Department of Mathematics

University of California

Santa Barbara, CA 93106-3080 cooper@math.ucsb.edu

Jiang-Hua $\mathrm{Lu}$

Department of Mathematics

The University of Hong Kong

Pokfulam Rd., Hong Kong jhlu@maths.hku.hk
Don Blasius

Department of Mathematics University of California

Los Angeles, CA 90095-1555

blasius@math.ucla.edu

Robert Finn

Department of Mathematics Stanford University

Stanford, CA 94305-2125

finn@math.stanford.edu

Sorin Popa

Department of Mathematics

University of California

Los Angeles, CA 90095-1555

popa@math.ucla.edu

Paul Yang

Department of Mathematics

Princeton University

Princeton NJ 08544-1000

yang@math.princeton.edu

\section{PRODUCTION}

Silvio Levy, Scientific Editor, production@msp.org

\section{SUPPORTING INSTITUTIONS}

ACADEMIA SINICA, TAIPEI

CALIFORNIA INST. OF TECHNOLOGY

INST. DE MATEMÁTICA PURA E APLICADA

KEIO UNIVERSITY

MATH. SCIENCES RESEARCH INSTITUTE

NEW MEXICO STATE UNIV.

OREGON STATE UNIV.

\author{
STANFORD UNIVERSITY \\ UNIV. OF BRITISH COLUMBIA \\ UNIV. OF CALIFORNIA, BERKELEY \\ UNIV. OF CALIFORNIA, DAVIS \\ UNIV. OF CALIFORNIA, LOS ANGELES \\ UNIV. OF CALIFORNIA, RIVERSIDE \\ UNIV. OF CALIFORNIA, SAN DIEGO \\ UNIV. OF CALIF., SANTA BARBARA
}

\author{
Vyjayanthi Chari \\ Department of Mathematics \\ University of California \\ Riverside, CA 92521-0135 \\ chari@math.ucr.edu \\ Kefeng Liu \\ Department of Mathematics \\ University of California \\ Los Angeles, CA 90095-1555 \\ liu@math.ucla.edu \\ Jie Qing \\ Department of Mathematics \\ University of California \\ Santa Cruz, CA 95064 \\ qing@cats.ucsc.edu
}

These supporting institutions contribute to the cost of publication of this Journal, but they are not owners or publishers and have no responsibility for its contents or policies.

See inside back cover or msp.org/pjm for submission instructions.

The subscription price for 2013 is US \$400/year for the electronic version, and \$485/year for print and electronic.

Subscriptions, requests for back issues and changes of subscribers address should be sent to Pacific Journal of Mathematics, P.O. Box 4163, Berkeley, CA 94704-0163, U.S.A. The Pacific Journal of Mathematics is indexed by Mathematical Reviews, Zentralblatt MATH, PASCAL CNRS Index, Referativnyi Zhurnal, Current Mathematical Publications and the Science Citation Index.

The Pacific Journal of Mathematics (ISSN 0030-8730) at the University of California, c/o Department of Mathematics, 798 Evans Hall \#3840, Berkeley, CA 94720-3840, is published monthly except July and August. Periodical rate postage paid at Berkeley, CA 94704, and additional mailing offices. POSTMASTER: send address changes to Pacific Journal of Mathematics, P.O. Box 4163, Berkeley, CA 94704-0163.

PJM peer review and production are managed by EditFLOW ${ }^{\circledR}$ from Mathematical Sciences Publishers.

PUBLISHED BY

mathematical sciences publishers

nonprofit scientific publishing

http://msp.org/

(C) 2013 Mathematical Sciences Publishers 


\section{PACIFIC JOURNAL OF MATHEMATICS}

Volume $264 \quad$ No. $1 \quad$ July 2013

On the center of fusion categories

ALAIN BRUGUIÈRES and ALEXIS VIRELIZIER

Connected quandles associated with pointed abelian groups

W. EdWin Clark, MoHamed ElHamdadi, Xiang-DONG HoU,

MASAHICO SAITO and TIMOTHY YEATMAN

Entropy and lowest eigenvalue on evolving manifolds

HongXin GuO, ROBERT PHILIPOWSKI and ANTON THALMAIER

Poles of certain residual Eisenstein series of classical groups

DIHUA JIANG, BAIYING LIU and LEI ZHANG

Harmonic maps on domains with piecewise Lipschitz continuous metrics 125

HAIGANG LI and CHANGYOU WANG

$q$-hypergeometric double sums as mock theta functions

JEREMY LOVEJOY and ROBERT OSBURN

Monic representations and Gorenstein-projective modules

XIU-HuA LUO and PU ZHANG

Helicoidal flat surfaces in hyperbolic 3-space

Antonio Martínez, JoÃo Paulo dos Santos and Keti

TENENBLAT

On a Galois connection between the subfield lattice and the multiplicative subgroup lattice

JOHN K. MCVEY

Some characterizations of Campanato spaces via commutators on Morrey 221 spaces

Shaoguang Shi and Shanzhen Lu

The Siegel-Weil formula for unitary groups 